\title{
Blogósfera periodística en Ecuador La opinión deliberada ¿Quiénes son y qué escriben?*
}

\author{
Punin Ma. Isabel ${ }^{* *}$ \\ Gutiérrez Benazir**
}

Recibido: 2015-07-26 Enviado a pares: 2015-07-30

Aprobado por pares: 2015-08-30 Aceptado: 2015-09-04

\section{Resumen}

El uso de blog por los periodistas ecuatorianos es un proceso relativamente nuevo; es utilizado como una alternativa de comunicación libre y vía de escape de las presiones internas y externas que existen en el sector de medios en el país desde la aprobación de la Ley de Comunicación.

Este estudio evidencia la existencia de 91 blogs divididos en tres categorías: 64 blogs personales alimentados por periodistas, 24 asociados a los grandes medios impresos en Ecuador y 3 blogs grupales dedicados a la distribución de noticias.

Se analizan los lugares de procedencia de los blogs y su relación con los niveles de acceso a la Red, temas tratados, la difusión que tienen en Facebook y Twitter, los tipos de blogs (personales, adjuntos a medios y grupales) y, por último, su índice de abandono y mortandad, usando la técnica de rastreo digital de evidencias (Roger, 2009).

En Ecuador los blogs no son monotemáticos; tratan varias ramas siendo el periodismo, la comunicación y la política los temas preferidos.

Palabras clave: periodismo, opinión, blogs, Ecuador, blogosfera, periodistas, comunicación.

* El presente artículo forma parte de los resultados de investigación del Proyecto de Investigación Nacional denominado: "Historia de la Comunicación de Ecuador. Prensa, Radio, TV y medios digitales" que financia la UT"PL desde el 2021

** PhD. En Comunicación y Periodismo por la Universidad Santiago de Compostela (España). Profesora tiempo completo en la Universidad Técnica Particular de Loja (Ecuador). Universidad Técnica Particular de Loja-Ecuador. mipunin@utpl.edu.ec

*** Becaria de investigación y responsabilidad universitaria (BRU). Proyecto "Historia de la Comunicación". Estudiante de la titulación en Comunicación Social de la Universidad Técnica Particular de Loja (Ecuador). Universidad Técnica Particular de Loja- Ecuador. bcgutierrezl@utpl.edu.ec 


\title{
Journalist blogosphere in Ecuador The deliberated opinion ¿W/ho are they and what do they write?
}

\begin{abstract}
The use of blogs by Ecuadorian journalist is a process relatively new, it's used like an alternative of free communication; way of escape form internal and external pressures that exist in the media sector of the country since the approbation of Communications Law.

This paper evidences the existence of 91 blogs divided in three categories: 64 personal blogs fed by journalist, 24 associated to the great print media in Ecuador, and 3 group ones dedicated to news feed.

There have been analyzed the blog's provenance places and it's relation with Network access levels, treated themes, the diffusion that it have in Facebook and Twitter, the kind of blogs (personal, attached to media and group ones) and, for the last, its index of abandonment and mortality, using digital tracking technique (Roger, 2009).

In Ecuador the blogs aren't monothematic; treat some branches, being journalism, communication and politics the preferred themes.
\end{abstract}

Key words: Journalism, opinion, blogs, Ecuador, blogosphere, journalist, communication.

\section{A Blogosfera periodística no Equador A opinião deliberada , ¿Quem são e o que escrevem?}

\section{Resumo}

O uso do blog pelos periodistas equatorianos é um processo relativamente novo; é utilizado como uma alternativa na comunicação livre e via de escape das pressões internas e externas que existem no setor dos meios no país desde a aprovação da Lei de Comunicação.

Este estudio evidência a existência de 91 blogues divididos em 3 categorias: 64 blogues pessoais que são de periodistas, 24 associados aos grandes meios impressos no Equador e 3 blogues grupais dedicados à distribuição de notícias.

Analisam-se os locais de procedência dos blogs e sua relação com os níveis de acesso à rede, tópicos, a difusão que tem no Facebook e no Twitter, os tipos de blogs (pessoais, anexos, a meios e grupais) e pelo último seu índice de abandono e mortalidade mediante o uso da técnica de rastreio digital de evidências (Roger, 2009).

No Equador, os blogs não são mono temáticos; têm várias ramas sendo o periodismo, a comunicação e a política como tópicos prediletos.

Palavras-chave: jornalismo, opinião, blogs, Equador, blogosfera, jornalistas, comunicação. 


\section{Introducción}

Sáez (2005) y Parra (2009) señalan que un blog es un espacio en la Red cuya razón de ser es servir de soporte para la escritura; un sitio donde se copilan notas de uno o más autores a modo de diario personal y tiene varias ventajas como contenido actualizable, realimentación de los internautas y vínculos con otros blogs, lo que les permite recibir las visitas procedentes de los enlaces en otras bitácoras. Los motivos que inducen a crear un blog son autoexpresión, deseo de contacto, informar e informarse, y labrar una reputación en el entorno web.

Sáez (2005), citando la entrevista que el diario El País hizo el 23 de marzo de 2005 al escritor y en su día padre del antiguo "nuevo" periodismo, Tom Wolfe, destaca lo siguiente:

Los blogs son probablemente algo bueno. Seguramente, es lo mejor que le ha ocurrido al periodismo. La mayoría de los blogs son una basura, pero si se busca, siempre se puede encontrar algo interesante. Los blogs pueden reproducir rumores que la prensa no publica, son fuentes posibles que nos dan una información que no teníamos antes (p. 16).

Los blogs y las redes sociales no han sustituido al periodismo, al contrario, han influido profundamente sobre él. Internet funciona como campo de investigación, plataforma de distribución de contenidos y contacto con fuentes alternativas (Diezhandino, 2012), una relación complementaria que evoluciona (Fogel; Patiño, 2008).

Las primeras generaciones de blogueros ecuatorianos están marcada por la Net Generation o los "nativos digitales", que nacieron, o vivieron su juventud en la época en la que Internet se popularizó y pasó de la web 1, en la cual solo eran receptores de servicios, a la Web 2.0, donde participan, intercambian opiniones y colaboran en el desarrollo de la tecnología, e instintivamente tuvieron facilidad al aprender a manejarla; en este grupo están todos los niños, adolescentes y jóvenes que en 1999 tenían entre 2 y 22 años (Ovelar, Benito \& Romo, 2009; García \& Núñez, 2009).

La principal diferencia entre los nativos digitales y los inmigrantes digitales (aquellos nacidos antes de 1977) es su manera de procesar y acceder a la información, convirtiéndose la Internet, para los nativos, en su principal medio de expresión, identidad y relación (Rubio; 2010, citado por Frutos, Sánchez \& Vázquez, 2014); sin embargo, eso no garantiza que sepan cómo utilizarla correctamente, "esos contenidos que ponen a circular socialmente, estén construidos con responsabilidad y conociendo sus consecuencias" (Sandoval \& Aguaded, 2012: 15).

El uso del blog como herramienta de comunicación es un tema relativamente nuevo; se destacan los estudios de contenidos e impacto (Meso, 2009; Masip, 2010) o de redacción digital (Salaverria, 2005, Franco, 2009), entre otros

En el caso particular de Ecuador los antecedentes documentados por la Academia están relacionados con el proceso de constitución de la Asamblea Nacional Constituyente (2007) que marcó un hito en el uso del blog como espacio de intercambio y debate 
alternativo con los blogs personales de los asambleístas; en ellos brindaban su propia opinión de los temas que en las reuniones acontecían (Albonoz, 2010), pero, pese a ello, en el país no existe un censo exacto y actualizado de este instrumento de comunicación.

Las restricciones que impone la Ley Orgánica de Comunicación, en vigencia en Ecuador desde 2013, activó la presencia paralela de blogs vinculados con periodistas de referencia, pertenecientes en su mayoría a medios de comunicación privados, logrando identificar espacios de comunicación liderados por reporteros ecuatorianos en diferentes plataformas en los cuales pueden opinar con mayor libertad.

Freedom House, entidad que vigila por la libertad de expresión de todos los países del mundo, declara lo siguiente: "En menos de cinco años desde su toma del poder, el Presidente Rafael Correa ha convertido Ecuador en uno de los países con más restricciones sobre la libertad de expresión en América Latina y ha tomado pasos hacia el ataque a la libertad de asociación" (Freedom House, 2015), lo cual explica perfectamente la existencia del fenómeno blog en Ecuador.

Este portal (Freedom House) califica la libertad de expresión en la Red, y a Ecuador le ha asignado una calificación de 37/100, siendo 0 la mejor puntuación y 100 la peor. Para obtener estos 37 puntos se evalúan tres parámetros:

1. Obstáculos de acceso: facilidad con la cual los ecuatorianos se conectan a la red, Ecuador posee una valoración de 9/25, siendo 0 el mejor y 25 el peor. Mejoró un punto en comparación a 2013 que poseía 10/25.

2. Límites de Contenido: censura en redes, aquí Ecuador posee una nota de 11/25, manteniéndose en la misma calificación que tuvo en 2013.

3. Violaciones a los derechos de los usuarios: o persecución en línea, hackeos y demás atropellos virtuales. En este punto Ecuador posee una puntuación de 17/40, habiendo empeorado un punto en comparación a 2013, que se ubicaba en 16/40 (Freedom House, 2014)'.

\section{Metodología}

La presente investigación realiza un inventario detallado de los blogs ecuatorianos que generan debate en torno al ejercicio periodístico, la comunicación y los medios en Ecuador, usando como técnica de investigación el rastreo digital de datos (Rogers, 2009) y el almacenamiento digital (Howinson; WigginsE Growten,2011).

En la realización del inventario participó un promedio de 100 estudiantes, matriculados en la materia de Géneros Periodísticos de la Titulación en Comunicación Social de la Universidad Técnica Particular de Loja en Modalidad Abierta, residentes a lo largo y ancho del país. El rastreo digital y la documentación de evidencias se realizaron entre abril y agosto del 2014.

1 Este estudio se publica en mayo de cada año, por eso no se han podido obtener datos actualizados de finales de 2014 y principios de 2015. 
Se incluyó la clasificación en un promedio de 91 blogs, divididos en 25 categorías, que representan el total del universo seleccionado para este estudio. Se estudia el número de seguidores que tienen en las redes sociales más populares en Ecuador: Facebook y Twitter.

\section{Perfil topográfico de la blogosfera periodística en Ecuador}

"La blogosfera es una innovación del espacio virtual que ha logrado abordar casi todos los aspectos sociales de Red" (Albornoz, 2010: 67) que, en el caso particular de Ecuador, inició con muy buen aliento, pero en el transcurso del tiempo, y desde el auge en el uso masivo de las redes sociales en 2009, ha ido perdiendo influencia y posición como parte de la esfera pública ecuatoriana, como lo evidenciaremos más adelante.

El fenómeno blog periodístico tiene su cobertura nacional, aunque se evidencia una concentración mayor en las capitales de provincia, Quito (41) y Guayaquil (16) las dos ciudades principales del país, y con mejores niveles de vida y de acceso a la Red.

También existen algunos blogs que no registran ubicación física (12); aquí vale destacar el blog de Lola Cienfuegos. No se conoce su ubicación, ni su nombre y solo utiliza la red social Twitter muy rara vez. Según las propias versiones de la involucrada en una entrevista con El Telégrafo (2012) ${ }^{2}$ su cuenta de twitter y su blog han tenido intentos de hackeo. Dos cuentas de Twitter dicen ser la verdadera Lola: @lolacienfuegos y @Iolacienfuegos, a ello se suma su blog (https://lolacienfuegos.wordpress.com/).

Después de Quito, Guayaquil y las ubicaciones desconocidas, está Cuenca con 6 blogs, seguido de Manta con 3, Loja y Riobamba con 2 blogs cada una, y Naranjal e Imbabura, son las que poseen menos blogs por ciudad con uno en cada una.

La cantidad de blogs por ciudad coincide con el hecho de que entre más poblada es una provincia, mejor nivel de educación tiene y los niveles de acceso a la Red son mayores y el número de blogueros más alto está como Quito y Guayaquil; en cambio, si la ciudad es pequeña, su número decae, como por ejemplo en Naranjal e Ibarra.

Tabla 1. Promedio de habitantes, ingreso a internet, escolaridad y blogs por provincia

\begin{tabular}{lcccc}
\hline \multicolumn{1}{c}{ Ciudad } & Habitantes & $\begin{array}{c}\text { Promedio de } \\
\text { ingreso a Internet }\end{array}$ & $\begin{array}{c}\text { Nivel de educación (Promedio en } \\
\text { años de escolaridad) }\end{array}$ & Blogs \\
\hline Quito & 2.239 .191 & $48.2 \%$ & 11.1 & 34 \\
Guayaquil & 2.350 .915 & $28.8 \%$ & 10.5 & 14 \\
Cuenca & 505.585 & $40.9 \%$ & 10 & 6 \\
Manta & 226.477 & $27.6 \%$ & 9.8 & 3 \\
Loja & 214.855 & $40.4 \%$ & 10.9 & 2 \\
Riobamba & 225.741 & $36.6 \%$ & 10.1 & 2 \\
Ibarra & 181.175 & $37.2 \%$ & 9.8 & 1 \\
Naranjal & 69.012 & $9.1 \%$ & 7.6 & 1 \\
\hline
\end{tabular}

Fuente: INEC 2014

2 Entrevista a Lola Cienfuegos por "El Telégrafo": http://voto2013.eltelegrafo.com.ec/noticias/tecnologia/ item/lola-aquella-mujer-que-libra-en-twitter-una-batalla-a-cienfuegos.html el 25 de marzo de 2012. 
También existen cuatro blogs cuyos autores se encuentran radicados en el extranjero. Dos autores de blogs personales; Jessica Jiménez en Madrid, España con Vivencias de Migración (http://vivenciasdemigracion.blogspot.com/) y Milton Ramírez en Newark, New Jersey, Estados Unidos con su blog Spanish Readers Book (http://miltonramirez.org/), ambos autores tratan en sus post la migración y dan sus opiniones acerca de la situación de Ecuador y de los países de residencia.

Los otros dos son blogueros anexados a medios, uno vive en Colombia y otro en México; uno en Bogotá, Dennis Rodríguez, autor de PO-RUSSKII (http://www.elcomercio. com.ec/blogs/po-russkii) y Vuelta al Mundo en 80 Ideas (http://www.elcomercio.com.ec/blogs/ la-vuelta-al-mundo-en-80-ideas) que opina sobre temas nacionales e internacionales; y en Cuernavaca se radica Fausto Segovia Baus, educador y pedagogo, autor del blog de educación La silla vacía (http://www.elcomercio.com.ec/blogs/la-silla-vacia).

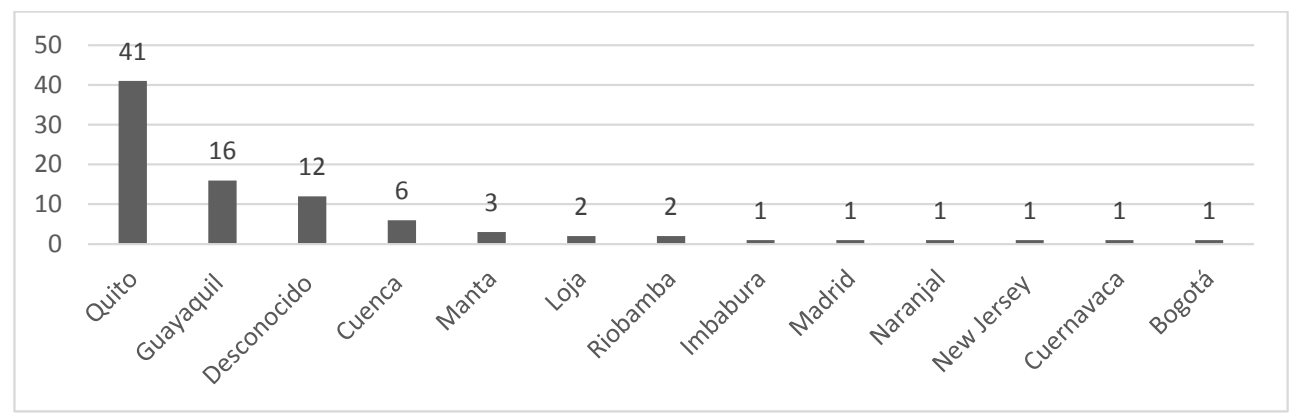

Gráfico 1. Blogs por ubicación geográfica

Fuente: elaboración propia

De los 91 blogs personales analizados en este trabajo, 60 pertenecen a hombres, 26 a mujeres, 3 son grupales y uno (¿Qué hay en el cine?) no tiene sexo definido ya que su autor es desconocido.

\section{Los principales temas tratados en los blogs ecuatorianos}

En Ecuador la mayoría de los blogs posee dos o tres categorías distintas en sus temas, rara vez se centran en uno solo. Para mejorar el proceso de visualización de las grandes temáticas de discusión las autoras plantearon las siguientes categorías de clasificación, que, a su vez, tiene relación con las temáticas más recurrentes de los blogs reportados por los estudiantes como los de mayor preferencia:

Periodismo (38), comunicación (29), política (21), opinión (15), tecnología (11), entretenimiento (8), deportes (5), humor (4), cine (3), relatos (4), fotografía (2), música (2), migración (2), medio ambiente (2) y al final feminismo, moda, historia, viajes, poesía, retail, aviación, maternidad, reflexiones, turismo y educación, con un blog por tema.

La clasificación temática ha sido amplia debido a la diversidad de los temas publicados; esto permite deducir la ausencia del blogs especializados en periodismo; por el 
contrario, los blogueros hacen uso de la transdisciplinaridad del periodismo, entendida como "un conocimiento relacional y complejo" (Morín, 2012). Esta polivalencia formativa fue impulsada por la CIESPAL (Centro Internacional de Estudios Superiores de Comunicación para América Latina) en la década del 60, y aún se mantiene en las aulas universitarias ecuatorianas.

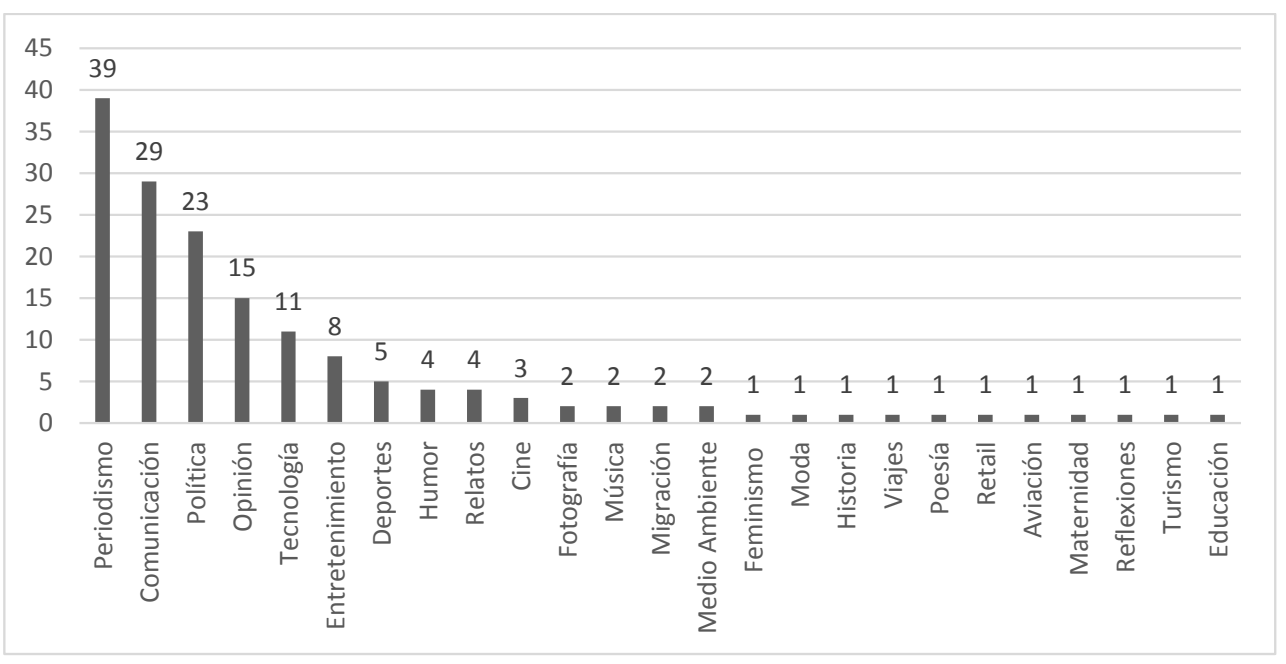

Gráfico 2. Temas tratados en los blogs de Ecuador

Fuente: elaboración propia

En los blogs que tratan el periodismo y la comunicación es en donde más borrosa se vuelve la línea entre lo periodístico y lo que no es periodístico ya que aparte de presentar reportajes y hechos (que es lo que llamaríamos periodismo "duro"), suelen redactarlos con un estilo propio -serio, sarcástico, humorístico, etc.-, pero también opinan y sacan a relucir ideas.

La política es uno de los temas habituales de los escritores en estudio; algunos están relacionados con los comentarios difundidos en los enlaces sabatinos que lidera el presidente R. Correa. Hay blogueros en contra de Rafael Correa como Carlos Andrés Vera (autor de Polificcion e hijo del activista político en contra de Correa, Carlos Vera. Actualmente retirado del activismo político en línea) y Víktor Vizcaíno (Autor de Plegaria Pagana y Víktor Vizcaíno) y blogueros, supuestamente a favor, como Lola Cienfuegos, sin embargo, ella misma ha señalado repetidas veces que "La Lola no está casada con nadie".

Los blogs de opinión en muchos casos no pasan de ser columnas que no se publican en medios impresos; por eso suelen mezclarse con periodismo, humor, política y otros temas.

Los blogs de humor a veces mezclan también opinión y periodismo, como Anota mi Nota (http://www.anotaminota.com/), blog en el que Blanca Moncada (@blankimonki), reportera de la sección ÁREA 51 Rock Show de diario Expreso habla de su propia vida, 
opina acerca de los temas de actualidad, o de cosas que ella ve y destaca en sus entradas, todas con un toque humorístico. También suele añadir entrevistas o reportajes entre sus post. Amelia la Loca (https://amelialaloca.wordpress.com/), Álter Ego de Evelyn Tapia (@ evytapiazurita), reportera de un periódico en Guayaquil, también utiliza el recurso del humor al hablar de su propia vida, sus reflexiones y sus pensamientos.

\section{Facebook y Twitter, medios de difusión}

"Las redes sociales, que no nacieron con fines periodísticos y que muchos profesionales de la información minusvaloraron en sus inicios" (López, Rodríguez \& Soengas, 2015) se han convertido en herramientas poderosas a la hora de difundir contenidos.

Facebook y Twitter son dos de las redes más populares en el Ecuador; Twitter cuenta con aproximadamente dos millones de cuentas en el país y Facebook con poco más de ocho millones de usuarios hasta enero de 2015 (Alcázar, 2015).

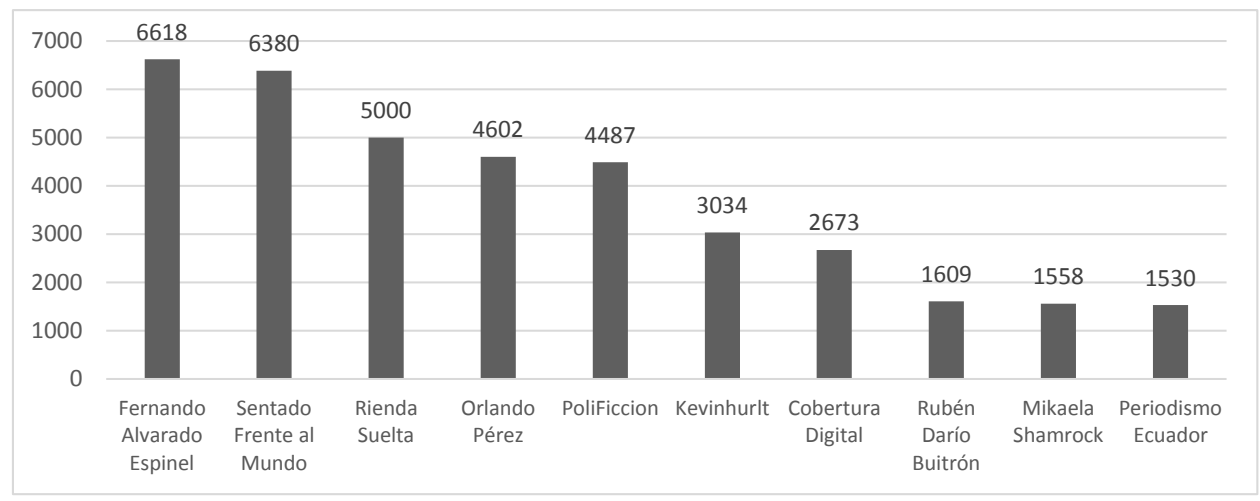

Gráfico 3. Blogs por popularidad de sus autores en Facebook*

Fuente: elaboración propia

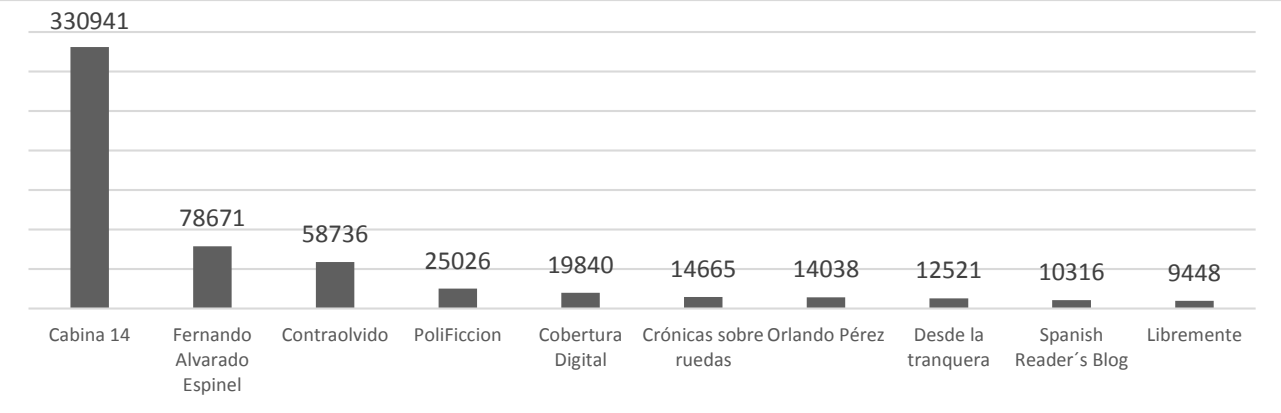

Gráfico 4. Blogs por la popularidad de sus autores en Twitter Fuente: elaboración propia

* Para esta tabla se utilizó el número de amigos de perfiles de los autores, ya que no todos los blogs no contaban con fanpages. 
La popularidad de los autores en Twitter no siempre se debe a sus blogs; por lo general se puede atribuir a méritos personales como la trayectoria profesional: entre los diez más populares tenemos a Diego Arcos, autor de Cabina 14, que supera por mucho al resto de blogueros en Twitter (330941 followers); esta diferencia se debe a su popularidad como presentador del programa deportivo TC Deportes y a su sección de opinión en la página de TC Televisión (http://www.tctelevision.com/tcdeportes/opinion/diego-arcos); le sigue Fernando Alvarado Espinel con su blog homónimo, director de la Secretaría Nacional de Comunicación (SECOM) ${ }^{3}$ con 78671 seguidores; luego está Diego Oquendo con Contraolvido, periodista de más de treinta años de trayectoria con 58736 seguidores; Carlos Andrés Vera, de Polificcion cineasta e hijo del activista político, ex presentador de televisión y candidato a presidente Carlos Vera con 25026 seguidores, seguido de Christian Espinoza con Cobertura Digital Experto en redes sociales, profesor universitario y conferencista de nuevos medios con 19840; Susana Morán Crónicas sobre ruedas, ganadora del concurso de Periodismo Digital de Fundamedios gracias a su reportaje en tiempo real acerca de la sublevación policial e intento de golpe de Estado a Rafael Correa del 30 de septiembre de 2011, con 14665; Orlando Pérez, director de El Telégrafo, con su blog homónimo Orlando Pérez tiene 14038 seguidores; Martín Pallares, Desde la Tranquera, otro periodista con 20 años de trayectoria, 12521; Milton Ramírez con Spanish Readers Blog, educador y también autor del blog "Education and Tech" (http://www.educationandtech.com/4), con 10316, y en décimo lugar, a Gabriela Calderón, autora de Libremente, articulista de El Universo e Investigadora de la ONG "El Cato", organización dedicada a promoción de políticas públicas acordes con la libertad individual en América Latina, con 9448 seguidores.

En el estudio de los Consultores de Comunicación Llorente y Cuenca: Mapa de poder en la Red: Influyentes online en Ecuador (2014) se presenta un gráfico con las 50 personas más populares en Twitter en el país. En este ranking aparece Fernando Alvarado Espinel, Director de la SECOM y autor del blog personal que lleva su nombre (http://fernandoalvaradoespinel.com/) en el puesto 19 con un aumento de popularidad subiendo del puesto 60 que ostentaba en 2013 y Diego Arcos, periodista deportivo y autor del blog Cabina 14 (http:// cabina14.blogspot.com/) en el puesto 34, habiendo tenido un descenso de popularidad, ya que en 2013 la misma lista lo ubicaba en el puesto 15. Orlando Pérez, director de El Telégrafo y autor del blog (http://orlandoperezsanchez.blogspot.com/) también aparece en este ranking, pero en un lugar bastante bajo; en 2013, se ubicaba en el puesto 113, pero en 2014 su popularidad bajó al 203. (Llorente \& Cuenca, 2014).

De los autores de los 90 blogs analizados, 27 no poseen cuenta en Facebook, y de los 64 blogs que sí tienen, doce son privados, no se puede acceder a estadísticas de amigos; en Twitter solo 19 no poseen red. La principal razón de esta diferencia es que los autores prefieren Facebook para relaciones personales y Twitter para difundir sus

3 La Secretaría Nacional de Comunicación (SECOM) es la organización encargada de asesorar al presidente Rafael Correa en materia comunicación e información

4 Education and Tech no fue incluido en esta investigación debido a que no está en español ni está dirigido a público ecuatoriano. 
contenidos, incluso el número de seguidores en Twitter en cada cuenta es aún mayor que el de Facebook.

\section{Tipos de blogs: personales, grupales y derivados del periodismo}

En Ecuador existen tres tipos de blogs definidos: los personales (64), en los que los autores expresan sus intereses, pensamientos y opiniones de los temas de actualidad; los derivados del periodismo (24), aquellos en los que el autor expresa sus opiniones pero que, sin embargo, están vinculados a algún medio de comunicación, y los grupales (3), en los que varias personas se reúnen para sacar un sitio adelante.

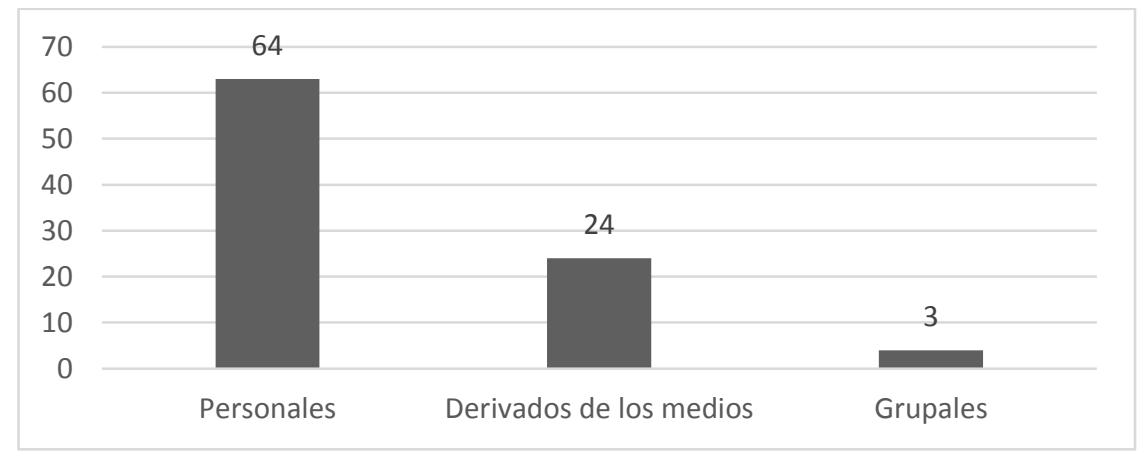

Gráfico 5. Número de blogs según su tipo

Fuente: elaboración propia

Los más numerosos son los personales: son más fáciles de mantener, no son una obligación, no existe censura de editores o jefes a la hora de publicar y son más un hobby que una responsabilidad. Aquí los periodistas se pueden tomar licencias a la hora de publicar una nota o sus pensamientos, pero aún en el espacio on-line están vigilados por las figuras de poder en Ecuador, las cuales sí aplican censura en el contenido publicado mediante la empresa Ares Rights, radicada en Madrid-España, que de cuando en cuando suele hacer que Twitter o Blogger bloqueen al usuario que infringió "derechos de autor" al publicar imágenes, vídeos o contenidos que aluden al presidente o al Gobierno sin que estas páginas puedan constatar si en verdad lo hizo debido a un vacío legal del cual esta empresa se aprovecha. Esta situación fue explicada desde varias perspectivas por varios blogueros: Lola Cienfuegos habló de esto en su entrada "Ares Rights y el cuento chino ¿Los giles, sus 'pobres víctimas'?" (https://lolacienfuegos.wordpress.com/2014/11/25/ares-rightsy-el-cuento-chino-los-giles-sus-pobres-victimas/) y Martín Pallares en su entrada "Ares Rights retira de la web aquello que incomoda al Gobierno" (http://www.elcomercio.com.ec/ blogs/desde-la-tranquera/ares-rights-retira-de-web.html) e incluso Fundamedios envió una carta pública a esta empresa y a Twitter pidiendo que se detenga el abuso en nombre de las leyes de derechos de autor (Fundamedios, 2014).

Un caso aparte fue el de Víktor Vizcaíno (@plegariapagana), autor de los blogs Plegaria Pagana (http://www.laplegariadeunpagano.com/) y Víktor Vizcaíno (http://www.viktor- 
vizcaino.blogspot.com/), el cual fue detenido en abril de 2011 por presuntas ofensas a Washington Pesantez, Fiscal General de la Nación, en la entrada "Washington Pesantez, criminal encubridor" (http://www.laplegariadeunpagano.com/2010/01/washingtonpesantezcriminalencubridor.html (El link se encuentra inactivo) de su blog Plegaria Pagana (Plan V, 2015). Su blog, Víktor Vizcaíno, fue hackeado en el mismo período de su detención, pero pudo recuperarlo en mayo al hackearlo de nuevo. En su entrada "Lo que natura no da..." (http://www.viktorvizcaino.blogspot.com/2011/05/lo-que-natura-no-da.html) rechaza la acción y acusa al gobierno de haberlo hecho; también habla de su detención y asegura que es la primera de muchas. En Plegaria Pagana no dedicó ningún post al hecho.

Tabla 2. Blogs personales en Ecuador

\begin{tabular}{|c|c|c|c|}
\hline Nombre del blog & Autor del blog & Dirección & Características básicas \\
\hline Cuencano en el planeta & Jorge Piedra Cardozo & $\begin{array}{l}\text { http://www.jorgepiedra.blogspot. } \\
\text { com/ }\end{array}$ & $\begin{array}{l}\text { Comunicación y entrete- } \\
\text { nimiento }\end{array}$ \\
\hline Periodismo por dentro & Ricardo Tello Carrión & $\begin{array}{l}\text { http://hablemosdeperiodismo. } \\
\text { blogspot.com/ }\end{array}$ & Periodismo \\
\hline Lunas Azules & Sylvana Tapia & http://www.lunasazules.com/ & Periodismo y feminismo \\
\hline La Columna quinta & $\begin{array}{l}\text { Juan Francisco Bel- } \\
\text { trán }\end{array}$ & $\begin{array}{l}\text { http://lacolumnaquinta.word- } \\
\text { press.com/ }\end{array}$ & Periodismo \\
\hline Crónicas Urbanas & Marcela Rangel & $\frac{\mathrm{http://personalescro-}}{\underline{\mathrm{nicas} \cdot \mathrm{blogspot} \cdot \mathrm{com}}}$ & $\begin{array}{l}\text { Comunicación y perio- } \\
\text { dismo }\end{array}$ \\
\hline Voces del Sur & $\begin{array}{l}\text { Pablo Arciniegas } \\
\text { Ávila }\end{array}$ & $\begin{array}{l}\text { http://resistenciadelsur.blogspot. } \\
\text { com/ }\end{array}$ & Política \\
\hline Cabina 14 & Diego Arcos & cabinal4.blogspot.com & Periodismo deportivo \\
\hline El gato que fuma & Diego Cifuentes & $\begin{array}{l}\text { http://gatoquefuma.blogspot. } \\
\text { com/ }\end{array}$ & Periodismo y fotografía \\
\hline Lola Cienfuegos & Lola Cienfuegos & $\begin{array}{l}\text { https://lolacienfuegos.wordpress. } \\
\text { com }\end{array}$ & Periodismo y política \\
\hline $\begin{array}{l}\text { ECUADOR: LITERA- } \\
\text { TURA, FOTOGRAFÍA, } \\
\text { PERIODISMO }\end{array}$ & Rowny Pulgar Noboa & www.rownypulgar.blogspot.com & Política \\
\hline Novela & David Guzmán & http://perrologico.blogspot.com/ & Relato \\
\hline Periodismo & Mariana Neira & $\begin{array}{l}\text { http://mariana-neira.blogspot. } \\
\text { com/ }\end{array}$ & Comunicación y Opinión \\
\hline Libremente & Gabriela Calderón & www.libremente.org/?author $=2$ & $\begin{array}{l}\text { Comunicación, Periodis- } \\
\text { mo y tecnología }\end{array}$ \\
\hline Asiam@Dhanna & $\begin{array}{l}\text { Danna Hanna Aven- } \\
\text { daño }\end{array}$ & http://chinabella.blogspot.com/ & Comunicación y Opinión \\
\hline $\begin{array}{l}\text { Periodismo en Meta- } \\
\text { morfosis }\end{array}$ & Fernando Astudillo & $\begin{array}{l}\text { www.periodismoenmetamorfo- } \\
\text { sis.blogspot.com }\end{array}$ & Opinión y relatos \\
\hline Plegaria Pagana & Víctor Vizcaíno & $\begin{array}{l}\text { http://www.laplegariadeunpaga- } \\
\text { no.com/ }\end{array}$ & Periodismo y política \\
\hline Viktor Vizcaíno & Víctor Vizcaíno & $\begin{array}{l}\text { http://www.viktorvizcaino.blogs- } \\
\text { pot.com/ }\end{array}$ & Periodismo y política \\
\hline El diario de Juzz & Justin Pincay & Eldiariodejuzz.blogspot.com & Política \\
\hline
\end{tabular}




\begin{tabular}{|c|c|c|c|}
\hline Nombre del blog & Autor del blog & Dirección & Características básicas \\
\hline $\begin{array}{l}\text { Divagaciones no tan } \\
\text { vagas }\end{array}$ & Andersson Boscán & http://aboscan.blogspot.com/ & $\begin{array}{l}\text { Comunicación y entrete- } \\
\text { nimiento }\end{array}$ \\
\hline Anota mi nota & Blanca Moncada & www.anotaminota.com & Periodismo y humor \\
\hline $\begin{array}{l}\text { Ecuador Blondies Fas- } \\
\text { hion }\end{array}$ & Estefanía Sotomayor & $\begin{array}{l}\text { http://ecuadorblondiesfashion. } \\
\text { com/ }\end{array}$ & Periodismo y política \\
\hline Dr. Tecno & Jaime Freire & http://www.doctortecno.com/ & Moda y comunicación \\
\hline Mikaela Shamrock & Mishelle Macías & $\begin{array}{l}\text { https://mikaelashamrock.word- } \\
\text { press.com }\end{array}$ & $\begin{array}{l}\text { Comunicación y tecno- } \\
\text { logía }\end{array}$ \\
\hline Amelia la loca & Evelyn Tapia & $\begin{array}{l}\text { http://amelialaloca.wordpress. } \\
\text { com/ }\end{array}$ & $\begin{array}{l}\text { Comunicación y entrete- } \\
\text { nimiento }\end{array}$ \\
\hline Fotoplop & Braulio Orellana & http://fotoplop.blogspot.com/ & $\begin{array}{l}\text { Comunicación y entrete- } \\
\text { nimiento }\end{array}$ \\
\hline Periodismo Alternativo & Pedro López & $\begin{array}{l}\text { http://ecuadorperiodistico.blogs- } \\
\text { pot.com/ }\end{array}$ & Periodismo y política \\
\hline La cueva de Saulo & Saulo Ariel & $\begin{array}{l}\text { http://www.sauloarielibrexpre- } \\
\text { sion.blogspot.com }\end{array}$ & Periodismo \\
\hline $\begin{array}{l}\text { Gestión en comuni- } \\
\text { cación: investigación } \\
\text { aplicada, extensión y } \\
\text { servicios. }\end{array}$ & Abel Suing & https://abelsuing.wordpress.com/ & Opinión de temas varios \\
\hline Contacto Deportivo & Javier Vire Riascos & $\begin{array}{l}\text { http://www.javiervireriascos. } \\
\text { blogspot.com/ }\end{array}$ & Migración \\
\hline Vivencias de migración & Jessica Jiménez & $\begin{array}{l}\text { http://vivenciasdemigracion. } \\
\text { blogspot.com/ }\end{array}$ & $\begin{array}{l}\text { Comunicación y perio- } \\
\text { dismo }\end{array}$ \\
\hline Juan Pablo Trampúz & Juan Pablo Trampúz & $\begin{array}{l}\text { www.juanpablotrampuz.word- } \\
\text { press.com }\end{array}$ & $\begin{array}{l}\text { Comunicación y entrete- } \\
\text { nimiento }\end{array}$ \\
\hline Viviana Palacios Z. & $\begin{array}{l}\text { Lisseth Viviana Pala- } \\
\text { cios Zambrano }\end{array}$ & vivianapalaciosz.blogspot.com & $\begin{array}{l}\text { Comunicación y entrete- } \\
\text { nimiento }\end{array}$ \\
\hline Los matices de Belén & Belén Salinas Castro & belsalinascastro.blogspot.com & Política y medio ambiente \\
\hline $\begin{array}{l}\text { Kevinhurlt/ Rafael Mén- } \\
\text { dez Meneses }\end{array}$ & $\begin{array}{l}\text { Rafael Méndez Me- } \\
\text { neses }\end{array}$ & http://kevinhurlt.blogspot.com & $\begin{array}{l}\text { Opinión de temas de EE. } \\
\text { UU. y Ecuador }\end{array}$ \\
\hline Spanish Reader's Blog & Milton Ramírez & $\begin{array}{l}\text { h t t p : // m i l t o n r a m i r e z } \\
\text { org/2004/11/17/veronica-garces- } \\
\text { y-diario-el-universo/ }\end{array}$ & Comunicación y Opinión \\
\hline $\begin{array}{l}\text { Fernando Alvarado } \\
\text { Espinel }\end{array}$ & Fernando Alvarado & $\begin{array}{l}\text { http://fernandoalvaradoespinel. } \\
\text { com/ }\end{array}$ & $\begin{array}{l}\text { Periodismo, Política y } \\
\text { opinión }\end{array}$ \\
\hline Contraolvido & Diego Oquendo & http://www.diegooquendo.com/ & $\begin{array}{l}\text { Periodismo, Política y me- } \\
\text { dio ambiente }\end{array}$ \\
\hline Polificcion & Carlos Andrés Vera & http://polificcion.wordpress.com/ & $\begin{array}{l}\text { Comunicación, Periodis- } \\
\text { mo y tecnología }\end{array}$ \\
\hline Cobertura Digital & Christian Espinoza & www.coberturadigital.com & $\begin{array}{l}\text { Comunicación y tecno- } \\
\text { logía }\end{array}$ \\
\hline Internet en Ecuador & Christian Espinoza & $\begin{array}{l}\text { http://www.internetenecuador. } \\
\text { blogspot.com/ }\end{array}$ & Comunicación y Opinión \\
\hline Crónicas sobre ruedas & Susana Morán & $\begin{array}{l}\text { http://cronicassobreruedas. } \\
\text { blogspot.com/ }\end{array}$ & $\begin{array}{l}\text { Comunicación y perio- } \\
\text { dismo }\end{array}$ \\
\hline Orlando Pérez & Orlando Pérez & $\begin{array}{l}\text { http://orlandoperezsanchez. } \\
\text { blogspot.com/ }\end{array}$ & Periodismo y política \\
\hline
\end{tabular}


Blogósfera periodística en Ecuador La opinión deliberada ¿Quiénes son y qué escriben?

\begin{tabular}{|c|c|c|c|}
\hline Nombre del blog & Autor del blog & Dirección & Características básicas \\
\hline $\begin{array}{l}\text { Periodismo desde mis } \\
\text { ojos }\end{array}$ & Luis Vivanco & $\begin{array}{l}\text { www.periodismodesdemisojos. } \\
\text { blogspot.com }\end{array}$ & $\begin{array}{l}\text { Comunicación y tecno- } \\
\text { logía }\end{array}$ \\
\hline Ciudad@City & $\begin{array}{l}\text { María Cristina Mar- } \\
\text { tínez }\end{array}$ & $\begin{array}{l}\text { http://srtamartinez.blogspot. } \\
\text { com/ }\end{array}$ & Historia, comunicación \\
\hline $\begin{array}{l}\text { Sentado Frente al Mun- } \\
\text { do }\end{array}$ & Carlos Suasnavas & $\begin{array}{l}\text { http://www.sentadofrentealmun- } \\
\text { do.com/ }\end{array}$ & $\begin{array}{l}\text { Viajes alrededor del mun- } \\
\text { do }\end{array}$ \\
\hline www.estebanavila.com & Esteban Ávila & www.estebanavila.com & $\begin{array}{l}\text { Comunicación, Periodis- } \\
\text { mo y tecnología }\end{array}$ \\
\hline Bizness is Bizness & Albertina Navas & $\begin{array}{l}\text { http://www.biznessisbizness. } \\
\text { com/ }\end{array}$ & Periodismo deportivo \\
\hline Todo se borró & Silvia Azul & $\begin{array}{l}\text { http://todoseborro.blogspot. } \\
\text { com/ }\end{array}$ & $\begin{array}{l}\text { Comunicación y tecno- } \\
\text { logía }\end{array}$ \\
\hline Rafael Bonifaz & Rafael Bonifaz & http://rafael.bonifaz.ec/blog/ & Política y cine \\
\hline $\begin{array}{l}\text { Libros, autores y ries - } \\
\text { gos }\end{array}$ & Eduardo Varas & $\begin{array}{l}\text { https://eduardovarasc.wordpress. } \\
\text { com/ }\end{array}$ & Periodismo y política \\
\hline Rienda Suelta & Hernán Ramos & $\begin{array}{l}\text { http://ramoshernan.blogspot. } \\
\text { com/ }\end{array}$ & $\begin{array}{l}\text { Comunicación, Periodis- } \\
\text { mo y tecnología }\end{array}$ \\
\hline TIKINAUTA & José Rivera Costales & www.tikinauta.com/blog & Periodismo y política \\
\hline Mundo Mamá & Paulina Escobar & $\begin{array}{l}\text { https://medium.com/mundo- } \\
\text { mama }\end{array}$ & Opinión \\
\hline Estado de Propaganda & Roberto Aguilar & http://estadodepropaganda.com/ & Periodismo y política \\
\hline $\begin{array}{l}\text { El lado oscuro... ¿del } \\
\text { periodismo? }\end{array}$ & Verónica Miño & $\begin{array}{l}\text { http://veromynoticias.blogspot. } \\
\text { com }\end{array}$ & Comunicación política \\
\hline $\begin{array}{l}\text { Rostro Adusto: blog de } \\
\text { Gustavo Abad. }\end{array}$ & Gustavo Abad. & $\begin{array}{l}\text { http://rostroadusto.blogspot. } \\
\text { com/ }\end{array}$ & $\begin{array}{l}\text { Comunicación, periodis- } \\
\text { mo y tecnología }\end{array}$ \\
\hline La palabra abierta & Leonardo Parrini & $\begin{array}{l}\text { http://lapalabrabierta.blogspot. } \\
\text { com/ }\end{array}$ & Periodismo y política \\
\hline Rubén Darío Buitrón & Rubén Darío Buitrón & $\begin{array}{l}\text { http://rubendariobuitron.word- } \\
\text { press.com/ }\end{array}$ & $\begin{array}{l}\text { Periodismo y medio am- } \\
\text { biente }\end{array}$ \\
\hline Andrés Reliche & Andrés Reliche & http://reliche.blogspot.com/ & Relatos y poesía \\
\hline $\begin{array}{l}\text { Periódicos Digitales en } \\
\text { Ecuador y su a nivel } \\
\text { mundial }\end{array}$ & Clary & http://notidigitales.blogspot.com & Comunicación y opinión \\
\hline $\begin{array}{l}\text { Comunicación y opi- } \\
\text { nión }\end{array}$ & Francisco Garcés & $\begin{array}{l}\text { http://comunicacionyopinion. } \\
\text { blogia.com/ }\end{array}$ & $\begin{array}{l}\text { Comunicación, Periodis- } \\
\text { mo y tecnología }\end{array}$ \\
\hline Mujer en tierra firme & $\begin{array}{l}\text { Carla Badillo Coro - } \\
\text { nado }\end{array}$ & $\begin{array}{l}\text { http://mujerentierrafirme.blogs- } \\
\text { pot.com/ }\end{array}$ & Periodismo y humor \\
\hline Palabra independiente & Guido Moreno & $\begin{array}{l}\text { http://palabraindependiente. } \\
\text { blogspot.com }\end{array}$ & Periodismo y política \\
\hline Vilma & Vilma Vargas & $\begin{array}{l}\text { http://www.suplementoelpolvo- } \\
\text { rin.blogspot.com/ }\end{array}$ & Humor \\
\hline
\end{tabular}

Fuente: elaboración propia

Acerca de los blogs adscritos a los medios en Ecuador, muchos no son más que columnas de opinión con la etiqueta de "blog". En el diario El Universo existen 17 blogs adjuntos, de los cuales cuatro están vinculados, pero se alojan en páginas externas. Los alojados en el diario poseen pobre o nula hiper textualidad y no suelen poseer realimen- 
tación de los usuarios, ya que este periódico tiene los comentarios deshabilitados en su página. Casi todos están centrados en la opinión, excepto Modo Avión, enfocado en temas de aviación, 24/7 en maternidad, Vinilo y Bits, centrado en música, y retail -venta de objetos de consumo masivo- (El Retail y yo), por ejemplo.

El portal La República también posee blogs enlazados (6), los cuales se unen a él mediante vínculos en la parte inferior. Estos blogs, en comparación con los de El Comercio, si bien se enlazan con "La República" aparentan ser sitios web aparte con sus propios diseños, pero de estos blogs, uno está inactivo desde 2013 (¿Qué hay en el cine?) y se desconoce su autor y otro, Ráfagas, es de Carlos Andrés Vera, y está abandonado desde enero de 2014.

Tabla 3. Blogs adjuntos al medio "La República"

\begin{tabular}{|c|c|c|c|}
\hline Nombre & Autor & Dirección & Temática \\
\hline Animales sónicos & Gustavo Pérez & $\begin{array}{l}\text { http://www.larepublica.ec/animales- } \\
\text { sonicos/ }\end{array}$ & Música \\
\hline El blog de Catrina & Catrina & $\begin{array}{l}\text { http://www.larepublica.ec/blogde- } \\
\text { catrina/ }\end{array}$ & Reflexiones \\
\hline Mirada Global & $\begin{array}{l}\text { Luis Fernando } \\
\text { Ayala }\end{array}$ & $\begin{array}{l}\text { http://www.larepublica.ec/mirada- } \\
\text { global/ }\end{array}$ & Periodismo \\
\hline Ráfagas & $\begin{array}{l}\text { Carlos Andrés } \\
\text { Vera }\end{array}$ & http://www.larepublica.ec/rafagas/ & $\begin{array}{l}\text { Relatos, política y } \\
\text { opinión }\end{array}$ \\
\hline Tiempo libre & $\begin{array}{l}\text { Úrsula } \\
\text { Bresciani }\end{array}$ & $\begin{array}{l}\text { http://www.larepublica.ec/tiempo - } \\
\text { libre/ }\end{array}$ & Turismo \\
\hline $\begin{array}{l}\text { ¿Qué hay en el } \\
\text { cine? }\end{array}$ & Desconocido & $\begin{array}{l}\text { http://www.larepublica.ec/quehaye- } \\
\text { nelcine/ }\end{array}$ & Cine \\
\hline
\end{tabular}

Fuente: elaboración propia

Tabla 4. Blogs adjuntos al medio "El Comercio"

\begin{tabular}{|l|l|l|l|}
\hline \multicolumn{1}{|c|}{ Nombre del blog } & \multicolumn{1}{|c|}{ Autor } & \multicolumn{1}{|c|}{ Enlace } & \multicolumn{1}{c|}{ Temática } \\
\hline Desde la tranquera & Martin Pallares. & $\begin{array}{l}\text { http://www.elcomercio.com.ec/ } \\
\text { blogs/desde-la-tranquera }\end{array}$ & $\begin{array}{l}\text { Comunicación y } \\
\text { política }\end{array}$ \\
\hline Retrospectiva & $\begin{array}{l}\text { Carlos Rojas, } \\
\text { periodista }\end{array}$ & $\begin{array}{l}\text { http://www.elcomercio.com.ec/ } \\
\text { blogs/retrospectiva/ }\end{array}$ & $\begin{array}{l}\text { Periodismo y } \\
\text { política }\end{array}$ \\
\hline Pancho Cajas & Pancho Cajas & $\begin{array}{l}\text { http://panchocajascaricaturas. } \\
\text { blogspot.com/ }\end{array}$ & Periodismo y humor \\
\hline La silla vacía & $\begin{array}{l}\text { Fausto Segovia } \\
\text { Baus }\end{array}$ & $\begin{array}{l}\text { http://www.elcomercio.com.ec/ } \\
\text { blogs/la-silla-vacia }\end{array}$ & $\begin{array}{l}\text { Educación y } \\
\text { Opinión }\end{array}$ \\
\hline $\begin{array}{l}\text { Vuelta al mundo en } \\
80 \text { días }\end{array}$ & $\begin{array}{l}\text { Dennis } \\
\text { Rodríguez }\end{array}$ & $\begin{array}{l}\text { http://www.elcomercio.com.ec/ } \\
\text { blogs/la-vuelta-al-mundo-en- } \\
\text { 80-ideas }\end{array}$ & Opinión \\
\hline De taquito & Paola Gavilanes & $\begin{array}{l}\text { http://www.elcomercio.com.ec/ } \\
\text { blogs/de-taquito }\end{array}$ & Opinión y deportes \\
\hline
\end{tabular}


Blogósfera periodística en Ecuador La opinión deliberada ¿Quiénes son y qué escriben?

\begin{tabular}{|l|l|l|l|}
\hline \multicolumn{1}{|c|}{ Nombre del blog } & \multicolumn{1}{|c|}{ Autor } & \multicolumn{1}{|c|}{ Enlace } & \multicolumn{1}{c|}{ Temática } \\
\hline El guapo de la barra & $\begin{array}{l}\text { Alejandro } \\
\text { Ribadeneira }\end{array}$ & $\begin{array}{l}\text { http://www.elcomercio.com/blogs/ } \\
\text { el-guapo-de-la-barra }\end{array}$ & $\begin{array}{l}\text { Periodismo y } \\
\text { política }\end{array}$ \\
\hline Lanzando números & $\begin{array}{l}\text { César Augusto } \\
\text { Sosa }\end{array}$ & $\begin{array}{l}\text { https://lanzandonumeros.word- } \\
\text { press.com/ }\end{array}$ & $\begin{array}{l}\text { Periodismo y } \\
\text { economía }\end{array}$ \\
\hline Planeta Deporte & $\begin{array}{l}\text { Santiago } \\
\text { Guerrero }\end{array}$ & $\begin{array}{l}\text { http://www.elcomercio.com.ec/ } \\
\text { blogs/planeta-deporte }\end{array}$ & $\begin{array}{l}\text { Periodismo } \\
\text { deportivo }\end{array}$ \\
\hline Vinilo y Bits & Santiago Endara & https://viniloybits.wordpress.com/ & Música \\
\hline Guai Fai & Estéfano Dávila & $\begin{array}{l}\text { http://www.elcomercio.com.ec/ } \\
\text { blogs/guai-fai }\end{array}$ & Tecnología \\
\hline El Retail y Yo & $\begin{array}{l}\text { Berenice } \\
\text { Narváez }\end{array}$ & $\begin{array}{l}\text { http://berenicenarvaez.com/wor- } \\
\text { dpress/ }\end{array}$ & Retail \\
\hline Modo Avión & $\begin{array}{l}\text { Pedro } \\
\text { Maldonado }\end{array}$ & $\begin{array}{l}\text { http://www.elcomercio.com.ec/ } \\
\text { blogs/modo-avion }\end{array}$ & Aviación \\
\hline PO-RUSSKII & $\begin{array}{l}\text { Dennis } \\
\text { Rodríguez }\end{array}$ & $\begin{array}{l}\text { http://www.elcomercio.com.ec/ } \\
\text { blogs/po-russkii }\end{array}$ & Migración \\
\hline El foco al fotógrafo & Vicente Costales & $\begin{array}{l}\text { http://www.elcomercio.com.ec/ } \\
\text { blogs/el-foco-al-fotografo }\end{array}$ & Fotografía \\
\hline 24-7 & $\begin{array}{l}\text { Nancy } \\
\text { Verdezoto }\end{array}$ & $\begin{array}{l}\text { http://www.elcomercio.com.ec/ } \\
\text { blogs/24-7 }\end{array}$ & $\begin{array}{l}\text { Maternidad y } \\
\text { Opinión }\end{array}$ \\
\hline Letrometraje & Marcos Vaca & $\begin{array}{l}\text { http://www.elcomercio.com.ec/ } \\
\text { blogs/letrometraje }\end{array}$ & Cine y opinión \\
\hline
\end{tabular}

Fuente: elaboración propia

Los blogs grupales (3) normalmente funcionan como portales de noticias, excepto uno, el Libro bloguero, que es un portal de entretenimiento, Bitácora 593: El libro bloguero ecuatoriano (http://libroblogueroec.blogspot.com/) es una iniciativa de Blanca Moncada (@ blankimonki) que busca crear una antología del blog en Ecuador y publicar en un libro los post más destacados de los blogueros ecuatorianos. Actualmente el Libro Bloguero ya cuenta con página de Facebook (/libroblogueroec) y Twitter (@LibroBlogueroEC), y con todo un equipo de personas que manejan las redes sociales de este proyecto.

La Barra Espaciadora es una revista digital de periodismo literario, cuyo equipo está formado por nueve personas. Sus secciones son: Lo duro, que abarca investigación periodística; Tinta negra, perspectiva editorial de la revista; La papa caliente, que abarca temas de actualidad; La falla, que habla de situaciones que no deberían pasar; Visor, sección que presenta fotografía y audiovisuales; Entre nos presenta entrevistas; Escape, donde los autores ponen vivencias personales; Última Fila con reseñas varias, y Carajo, de videoclips.

Periodismo Ecuador (http://periodismoecuador.com/) es un portal de noticias sin fines de lucro en el cual cualquiera que desee colaborar puede hacerlo con solo enviar su nota a info@periodismoecuador.com. Se define como: "Una herramienta de expresión para su autor y para todos quienes quieran verter información u opinión sin restricción alguna. La Internet poco a poco se convertirá en el único medio de libre expresión en el mundo y en Ecuador de hoy es el único medio para expresarse libremente". 
Tabla 5. Blogs grupales en Ecuador.

\begin{tabular}{|l|l|l|l|}
\hline \multicolumn{1}{|c|}{ Blog } & \multicolumn{1}{|c|}{ Autores } & \multicolumn{1}{c|}{ Enlace } & \multicolumn{1}{c|}{ Temático } \\
\hline $\begin{array}{l}\text { Libro Bloguero } \\
\text { Ecuador }\end{array}$ & $\begin{array}{l}\text { Blanca Moncada } \\
\text { y otros bloggers }\end{array}$ & http://libroblogueroec.blogspot.com/ & Entretenimiento. \\
\hline $\begin{array}{l}\text { La barra } \\
\text { espaciadora }\end{array}$ & Nueve Personas & http://labarraespaciadora.com/ & $\begin{array}{l}\text { Comunicación y } \\
\text { entretenimiento. }\end{array}$ \\
\hline $\begin{array}{l}\text { Periodismo } \\
\text { Ecuador }\end{array}$ & Desconocido & www.periodismoecuador.com & Periodismo. \\
\hline
\end{tabular}

Fuente: elaboración propia

\section{Blogs: supresión y abandono}

Los blogs poseen dos desventajas bastante graves: el nivel de mortalidad es alto, muchos experimentan y abandonan su proyecto, y el más grave de todos es que en cualquier momento un blog puede ser eliminado por su autor, y su contenido (útil o no) desaparecerá por completo, imposibilitando su consulta en el futuro. (Sáez, 2005; Ovadia, 2010). En Ecuador los autores solo se limitan a abandonar sus blogs o a eliminar entradas "problemáticas".

"En enero de 2014 el activista político, director de cine y escritor Carlos Andrés Vera, quien posee el popular blog Polificcion fue objetivo en Twitter. Después de que su hijo de tres años fuera amenazado en redes por un representante de gobierno -según los informes- Vera anunció que abandonaría el activismo y el debate político en línea". (Freedom House, 2014). En su entrada "Lo que no se ve" (https://polificcion.wordpress.com/2014/02/17/ lo-que-no-se-ve/) aclara las razones de su retiro. Su último post en Polificcion fue el 25 de febrero de 2014, y en su blog Ráfagas, anexado a La República, (http://www.larepublica.ec/ rafagas/) el último post fue publicado el 28 de enero del mismo año.

Lola Cienfuegos es una bloguera que se ha retirado y vuelto varias veces al escenario virtual ecuatoriano. Por ejemplo, su primera despedida fue en junio de 2009 debido a la promesa personal de retirarse cuando tuviera mil comentarios en su blog de Blogspot, pero en octubre del mismo año regresó para abandonar por completo el dominio en 2010. Ha habido varias discusiones debido a su identidad, Fernando Alvarado Espinel cayó en la polémica ya que el Twitter de Lola compartía su IP (http://periodismoecuador. com/2013/01/29/ip-de-fernando-alvarado-coincide-con-de-lolacienfuegos/), pero no se pronunció acerca de los rumores. Lola tiene dos direcciones registradas, una en la que su última actividad se dio a raíz del ataque a Charlie Hebdo en 11 de enero de 2015 (https:// lolacienfuegos.wordpress.com/) y otra que está abandonada desde 2010 (http://lolacienfuegos.blogspot.com/).

Belén Salinas Castro también abandonó un blog, pero fue por motivos externos, repentinamente ella ya no pudo acceder a El Prisma de las Mil Caras (http://belensalinascastro.blogspot.com/) y le tocó abandonarlo para crear un nuevo proyecto: Los Matices de Belén (http://belsalinascastro.blogspot.com/). Gracias al Libro Bloguero Ecuatoriano ella pudo regresar a la "alta esfera" de los blogueros de Ecuador. En su primera entrada "Bienvenidos 
y bien idos" (http://belsalinascastro.blogspot.com/2015/01/bienvenidos-y-bien-idos.html) ella relata lo que pasó con su blog anterior.

Un tipo de blog que hasta ahora no se había visto era un blog literario; una blog novela en la cual las entradas fueran capítulos de un relato y los lectores pudieran interactuar con el autor, la historia y los personajes (Casciari, 2005); Perrológico (http://perrologico.blogspot. $\mathrm{com} /$ ) de David Guzmán pudo marcar un antes y un después, pero después de publicar toda la historia en el primer y único post, el blog quedó abandonado desde diciembre de 2005.

Rubén Darío Buitrón también se retiró de la escena bloguera ecuatoriana por un par de meses (marzo-mayo de 2013) debido al escándalo de una entrevista no realizada en vivo a Martín Caparrós, periodista y escritor argentino, publicada el diario Expreso, entrevista que le costó el puesto de editor en el diario, y por la cual se vio obligado a borrar su blog y su cuenta de Twitter (@periodismotdnp) debido al revuelo que causó; cuando regresó en mayo del 2013 empezó desde cero, borrando todas sus entradas anteriores, y publicó un post en el que explicaba y pedía disculpas por todo el mal entendido; "La implacable guerra conmigo mismo" (https://rubendariobuitron.wordpress.com/2013/05/19/la-guerra-contrami-mismo/) y creó una nueva cuenta de Twitter (@rd _ bui) que es la que actualmente usa.

El abandono o inactividad prolongada es común en blogs personales, pero los blogs anexados a los medios también lo presentan. Un ejemplo es el blog ¿Qué hay en cine? (http://www.larepublica.ec/quehayenelcine/), anexado a La República, el cual tenía una actividad intensa en redes, pero el 22 de mayo de 2013 el autor desconocido dejó de postear.

Algo que se puede confundir fácilmente con abandono es el hecho de que muchos de los blogs poseen sus últimas entradas en 2014; sin embargo, demuestran que después de haber tenido un periodo de posteo intenso, generalmente en los años 2007 o 2008, anteriores del auge de las redes sociales en Ecuador en 2009, tuvieron un descenso en publicaciones. En este período algunos llegaron a poner más de 100 entradas, pero drásticamente su cantidad de post empieza a bajar, incluso hay algunos como Jorge Piedra en su blog Cuencano en el planeta (http://www.jorgepiedra.blogspot.com/) que durante los últimos dos años (2013-2012) solo publicó dos entradas, después de haber publicado 103 en 2007 o como Diego Cifuentes en El gato que fuma (http://gatoquefuma.blogspot.com/), que en 2008 publicó 157 entradas y en 2014 bajó a 9. No se puede saber con certeza qué blogs están completamente desatendidos debido a este factor.

\section{Conclusiones}

Los blogs son nuevas plataformas para la difusión de la escritura, pero en contraparte puede desaparecer súbitamente información importante si el autor decide dar de baja el sitio. En Ecuador los autores no siempre eliminan sus blogs, pero muchos se limitan a postear una vez al año o dejarlos abandonados por tiempos indeterminados.

Existe una relación entre el número de habitantes, la calidad de educación y la facilidad de acceso a Internet con el número de blogueros por ciudad; por lo tanto, es muy complejo hablar de una desconcentración informativa. 
En Ecuador existen más hombres blogueros que mujeres, y su popularidad en redes se debe a méritos personales antes que a sus blogs.

Los blogs en Ecuador no se especializan en un solo tema, son pluritemáticos y la mayoría abarcan 2 y 3 categorías al mismo tiempo.

Los autores de los blogs utilizan mayoritariamente Twitter para publicar sus trabajos y acercarse a los lectores. Facebook es utilizado en menor medida y más para mantener relaciones personales.

\section{Referencias bibliográficas}

Albornoz, M. (2010, mayo). Blogs, artefactos y política. Íconos: Revista de ciencias sociales, 37, pp. 65-74. Recuperado el 9 de febrero de 2015. Disponible en http://revistas.flacsoandes.edu.ec/ iconos/article/view/409/395

Alcázar Juan (27 de enero de 2015). Formación Gerencial: Ranking y Estadísticas Redes Sociales Ecuador. Recuperado el 10 de marzo de 2015. Disponible en http://blog.formaciongerencial.com/2014/05/16/ ranking-redes-sociales-ecuador-mayo-2014/

Casciari Hernán (2005, octubre) El blog en la literatura: Un acercamiento estructural a la blog-novela en Telos, 65, 95-97. Recuperado el 28 de febrero de 2015. Disponible en http://dialnet.unirioja.es/ servlet/articulo?codigo $=1342569$

Diezhandino, M. (coord.) (2012) El periodista en la encrucijada. Barcelona: Ariel.

Fogel, J., y Patiño, B. (2008). La prensa sin Gutenberg. El periodismo en la era digital. Recuperado el 22 de octubre de 2015. Disponible en http://www.editorialtaurus.com/uploads/ficheros/libro/primeraspaginas/200705/primeras-paginas-prensa-sin-gutenberg.pdf

Franco, G. (2009). El impacto de las tecnologías digitales en el periodismo y la democracia en América Latina y el Caribe. Recuperado el 27 de octubre de 2015. Disponible en http:// www.scribd.com/fullscreen/1 15015252 ?access_key=keymp7qd6aqomu35ari906Eallow_ share $=$ trueEescape $=$ falseEview_mode $=$ scroll.

Freedom House (2015). Ecuador. Recuperado el 22 de marzo de 2015. Disponible en https:// freedomhouse.org/program/ecuador

Freedom House (mayo de 2014). Freedom on the net: Ecuador 2014. Recuperado el 19 de febrero de 2015. Disponible en https://freedomhouse.org/report/freedom-net/2014/ecuador

Frutos Torres, B., Sánchez Valle, M. y Vázquez Barrio, T. (2014): Perfiles de adolescentes on line y su comportamiento en el medio interactivo en Icono 14, 12, 374-397. Recuperado el 22 de marzo de 2015. Disponible en http://www.iconol4.net/ojs/index.php/iconol4/article/view/208/461

Fundación Andina para la Observación y el Estudio de Medios-FUNDAMEDIOS (2014). Carta de Fundamedios a Ares Rights por censurar opiniones críticas en Internet. Recuperado el 22 de marzo de 2015. Disponible en http://www.fundamedios.org/defensa-y-promocion/articulos/carta-de-fundamediosares-rights-por-censurar-opiniones-criticas-en

García Guardia, M.L., Núñez, P. (2009, enero) Los Bloggers y su influencia en la imagen de una marca en Icono 14, 12, 242-252. Recuperado el 28 de febrero de 2015. Disponible en http://www.iconol 4. net/ojs/index.php/iconol4/article/view/343 
Howison, J., Crowston K., E Wiggins A. (2011). Validity issues in the use of social network analysis with digital trace data en Journal of the Association for Information Systems, 12 (12), 1-63. Recuperado el 15 de octubre de 2015. Disponible en http://crowston.syr.edu/content/validity-issues-use-socialnetwork-analysis-digital-trace-data

Instituto Nacional de Estadísticas y Censos-INEC. (2014). Ecuador en cifras. Recuperado el 18 de marzo de 2015. Disponible en:http://www.ecuadorencifras.gob.ec/resultados/

López, Xosé; Rodríguez, Ana; Soengas, Xosé (2015). Estrategia de los diarios online de proximidad para las redes sociales: el caso de La Voz de Galicia. CISTI-Workshops.

Llorente \& Cuenca. (2014). Mapa de poder en la red: influyentes online en Ecuador. Recuperado el 29 de marzo de 2015. Disponible en http://www.mapadepoderecuador.com/

Morín, Edgar (2012). Qué es la transdisciplinariedad. Recuperado el 23 de marzo de 2015. Disponible en http://www.edgarmorin.org/que-es-transdisciplinariedad.html

Ovadía S. MLIS (2006, enero) The Need to Archive Blog Content en The Serials Librarian, 51 (1), 95-102. Recuperado el 28 de febrero de 2015. Disponible en http://dx.doi.org/10.1300/J123v51n01_06

Ovelar, R., Benito, M., Romo, J. (2009, enero) Nativos digitales y aprendizaje: una aproximación a la evolución de este concepto en Icono 14, 12, 31-53. Recuperado el 28 de febrero de 2015. Disponible en http://www.icono14.net/ojs/index.php/icono14/article/view/332/209

Parra Valcarce, D. (2009, octubre) El uso de los blogs entre los profesores de periodismo en España en Icono 14, 14, 84-102. Recuperado el 28 de febrero de 2015. Disponible en http://www.iconol 4. net/ojs/index.php/icono14/article/view/304/181

Plan V. (2015, 23 de febrero). La Ciberguerra de Correa. Recuperado el 22 de marzo de 2015. Disponible en http://www.planv.com.ec/investigacion/investigacion/la-ciberguerra-correa

Rogers, Curtis R. (2009). Social media, libraries and web 2.0. How American libraries are using new tools for public relations and to attract new users. Recuperado el 22 de octubre de 2015. Disponible en $<$ http://www. slideshare.net/crr29061/social-media-libraries-and-web-20-how-american-libraries-are-using-newtools-for-public-relations-and-to-attract-new-users $>$

Sáez Vacas, F. (2005, julio). La blogosfera: un vigoroso subespacio de comunicación en Internet en TELOS, 64, 14 17. Recuperado el 28 de febrero 2015. Disponible en http://telos.fundaciontelefonica.com/ telos/articulotribuna.asp@idarticulo=3Erev=64.html

Salaverría, R. (2005). Redacción periodística en Internet. Pamplona: EUNSA.

Salaverría, R., García-Avilés, J. E Masip, P. (2010). Concepto de convergencia periodística. En López, X.; Pereira, X. (Coords.). Convergencia digital. Reconfiguración de los medios de comunicación en España (41-64). Santiago de Compostela: Servicio Editorial de la Universidad de Santiago de Compostela.

Sandoval Romero, Y. y Aguaded Gómez, J.I. (2012, octubre) Nuevas audiencias, nuevas responsabilidades. La competencia mediática en la era de la convergencia digital en Icono 14, 10(3), 8-22. Recuperado el 28 de febrero de 2015. Disponible en http://www.icono14.net/ojs/index.php/icono14/article/ view/197/374 